\title{
Complex Number Theory without Imaginary Number (i)
}

\author{
Deepak Bhalchandra Gode \\ Directorate of Census Operations, Mumbai, India \\ Email: deepakm n4@rediffmail.com
}

Received 26 July 2014; revised 20 September 2014; accepted 23 October 2014

Copyright (C) 2014 by author and OALib.

This work is licensed under the Creative Commons Attribution International License (CC BY). http://creativecommons.org/licenses/by/4.0/

(C) (i) Open Access

\section{Abstract}

In this paper new method is introduced for complex numbers; this method does not include imaginary number " $i$ " but produces the same results that occur in Addition, Subtraction, Multiplication \& Division of complex numbers, also proof of Eluer Formula $e^{i \theta}$ and De Moivre theorem without using imaginary number " $i$ ". Furthermore placing the light on the square root of a negative number, the square root of a negative number is equal to the square root of the same positive real number but with an angle of 90 degree to real line. The intention is that there's nothing mystical about imaginary number " $i$ ". The square root of minus one is just as real as any other number. Complex numbers exists without imaginary number " $i$ ". This paper is limited to the results which are already established.

\section{Keywords}

Addition, Subtraction, Multiplication, Division, Eluer Formula, De Moivre Theorem, Square Root of Negative Number

Subject Areas: Mathematical Analysis, Mathematical Logic, Foundation of Mathematics

\section{Introduction}

An algebra of real number is not enough to solve the equations like $X^{2}+1=0$. To avoid this, concept of complex number was introduced; it is a combination of real numbers and imaginary number $i$, where $i$ is equal to square root of minus one $\sqrt{-1}$. Since then it was used to solve the equation which has no solution in real number theory and also established its use in different disciplines like electronics, quantum theory etc. But since $\sqrt{-1}$ has no exact value \& meaning, it is a fact that an injudicious use of this symbol often leads to mutually contradictory and absurd conclusions ([1], p. 1).

It is also observed that in graphical representation of complex number the point $i$ shown on $\mathrm{Y}$ axis has exactly the same altitude or length as number 1 or -1 of $\mathrm{X}$ axis has from origin. This implies that value of $i=1$ is a 
contradiction, since we don't know the exact value of square root of -1 (i.e. $i=\sqrt{-1}$ ). So it is better to have a number system which does not involve " $i$ " but gives all the benefits of complex number system.

Herewith complex number without imaginary number is being introduced; it is a combination of real numbers \& trigonometric functions i.e. $\cos (\theta), \sin (\theta)$. Any number in complex number theory without " $i$ " is represented by $a \cos (\theta)+b \sin (\theta)$ where $a \& b$ are real numbers and $\theta=\tan ^{-1}(a / b)$. It has direction and altitude/modulus same as complex number but without " $i$ ”. In graphical representation, $a \cos (\theta)$ represents $X$ axis and $b \sin (\theta)$ represents $\mathrm{Y}$ axis.

This is a simple graph, $\mathrm{X}$ axis represents combination of real number " $a$ " and $\cos (\theta)$, whereas, $\mathrm{Y}$ axis represents combination of real number " $b$ " and $\sin (\theta)$ where $\theta=\tan ^{-1}(a / b)$. Points shown in Figure 1 are explained below

\begin{tabular}{ccccc}
\hline Point on graph & $\begin{array}{c}\text { Here real number } \\
\text { " } a \text { " equal to }\end{array}$ & $\begin{array}{c}\text { Here real number } \\
\text { " } b \text { " equal to }\end{array}$ & $\begin{array}{c}\text { Angle " } \theta \text { " equal to } \\
\tan ^{-1}(a / b)\end{array}$ & $\begin{array}{c}\text { Corresponding complex number without } \\
\text { imaginary number " } i \text { " is }\end{array}$ \\
\hline$(0,3)$ & 0 & 3 & $90 \mathrm{deg}$ & $3 \sin (90)$ \\
$(1,1)$ & 1 & 1 & $45 \mathrm{deg}$ & $\cos (45)+\sin (45)$ \\
$(3,2)$ & 3 & 2 & $33.69007 \mathrm{deg}$ & $3 \cos (33.69007)+2 \sin (33.69007)$ \\
$(5,0)$ & 5 & 0 & $0 \mathrm{deg}$ & $5 \cos (0)$ \\
$(-1,-2)$ & -1 & -2 & $243.4349 \mathrm{deg}$ & $-1 \cos (243.4349)-2 \sin (243.4349)$ \\
\hline
\end{tabular}

Note: 1) Here onwards short form "CNWOI" is used in place of complex number without imaginary number $i$; 2) The value of angle $\theta$ is taken in degrees; 3) The $\cos (\theta) \& \sin (\theta)$ can have angle from 0 to 360 degrees; 4) The $\tan (\theta)$ can have angle from -180 to 180 degrees.

Every CNWOI is interlinked with its members i.e. the real numbers $a \& b$, the angle $\theta$ and the length $R$ from origin (i.e. radius or modulus).

Let $a+i b$ be any complex number then its equal CNWOI is

$$
a \cos (\theta)+b \sin (\theta) \text { where } \theta=\tan ^{-1}(a / b)
$$

Radius or Modulus $R$ : $R$ is calculated by putting values of $\cos \& \sin$ for $\theta=\tan ^{-1}(a / b)=\theta_{0}$ (some angle) in CNWOI i.e.

$$
R=a \cos \left(\theta_{0}\right)+b \sin \left(\theta_{0}\right)
$$

Example: For a point $(0,3)$ in above Figure $1, \theta=90$ deg so

$$
\begin{aligned}
& R=a \cos \left(\theta_{0}\right)+b \sin \left(\theta_{0}\right)=0 \times \cos (90)+3 \times \sin (90) \\
& R=0+3 \times 1=3
\end{aligned}
$$

This $R$ is same as modulus of complex number i.e. $R=\sqrt{a^{2}+b^{2}}$.

Calculated $R$ for points shown in Figure 1 is given in table

\begin{tabular}{|ccccc}
\hline Point on graph & $\begin{array}{c}\text { Here real number " } a \text { " } \\
\text { equal to }\end{array}$ & $\begin{array}{c}\text { Here real number " } b \text { " } \\
\text { equal to }\end{array}$ & And angle " $\theta$ " equal to & $R$ is equal to \\
\hline$(0,3)$ & 0 & 3 & 90 deg & 3 \\
$(1,1)$ & 1 & 1 & 45 deg & 1.414214 \\
$(3,2)$ & 3 & 2 & 33.69007 deg & 3.605551 \\
$(5,0)$ & 5 & 0 & 0 deg & 5 \\
$(-1,-2)$ & -1 & -2 & 243.4349 deg & 2.236068 \\
\hline
\end{tabular}

Coefficients $\boldsymbol{a} \& \boldsymbol{b}$ : If " $R$ ” and “ $\theta_{0}$ ” are given then coefficients $a \& b$ are equal to $a=R \cos \left(\theta_{0}\right) \& b=R \sin \left(\theta_{0}\right)$ ([1], pp. $11 \& 12$ and [2], p. 11).

Example: Suppose " $R$ " and " $\theta_{0}$ " are 2.236068 and 243.4349 deg respectively then

$$
\begin{aligned}
& a=2.236068 \cos (243.4349)=2.236068 \times(-0.44721)=-1 \\
& b=2.236068 \sin (243.4349)=2.236068 \times(-0.89443)=-2
\end{aligned}
$$




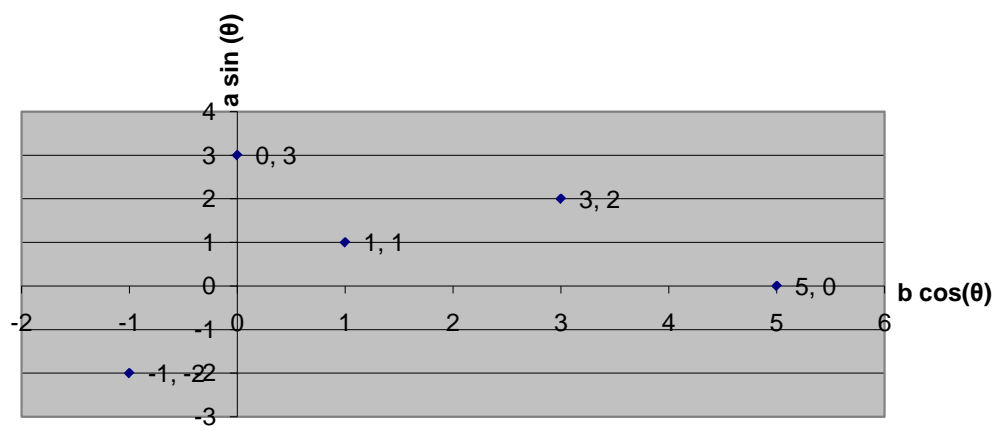

Figure 1. Graph of complex number without imaginary number “i”.

This is how " $R$ ", " $\theta$ ", " $a$ " \& " $b$ " are interlinked to each other.

\section{Addition of CNWOI}

There are two CNWOI

$$
a \cos \theta_{1}+b \sin \theta_{1} \text { where } \theta_{1}=\tan ^{-1}(a / b) \text { and } c \cos \theta_{2}+d \sin \theta_{2} \text { where } \theta_{2}=\tan ^{-1}(c / d)
$$

Then the addition is

$$
(a+c) \cos \theta_{3}+(b+d) \sin \theta_{3} \text { where } \theta_{3}=\tan ^{-1}((a+c) /(b+d))
$$

\section{Subtraction of CNWOI}

There are two CNWOI

$$
a \cos \theta_{1}+b \sin \theta_{1} \text { where } \theta_{1}=\tan ^{-1}(a / b) \text { and } c \cos \theta_{2}+d \sin \theta_{2} \text { where } \theta_{2}=\tan ^{-1}(c / d)
$$

Then the subtraction is

$$
(a-c) \cos \theta_{3}+(b-d) \sin \theta_{3} \text { where } \theta_{3}=\tan ^{-1}((a-c) /(b-d))
$$

\section{Multiplication of Two CNWOI}

There are two CNWOI

$$
a \cos \theta_{1}+b \sin \theta_{1} \text { where } \theta_{1}=\tan ^{-1}(a / b) \text { and } c \cos \theta_{2}+d \sin \theta_{2} \text { where } \theta_{2}=\tan ^{-1}(c / d)
$$

Putting angle of $\theta_{1}$ in first number will gate a number which is equal to $\sqrt{a^{2}+b^{2}}$ let's say this number is $R_{1}$, similarly putting angle of $\theta_{2}$ in second number will gate a number which is equal to $\sqrt{c^{2}+d^{2}}$ let's say this number $R_{2}$ then multiplication of this number is equal to

$$
\begin{gathered}
{\left[R_{1} R_{2} \cos \left(\theta_{1}+\theta_{2}\right)\right] \cos \theta_{3}+\left[R_{1} R_{2} \sin \left(\theta_{1}+\theta_{2}\right)\right] \sin \theta_{3}} \\
\text { where } \theta_{3}=\tan ^{-1}\left(\left[R_{1} R_{2} \cos \left(\theta_{1}+\theta_{2}\right)\right] /\left[R_{1} R_{2} \sin \left(\theta_{1}+\theta_{2}\right)\right]\right)
\end{gathered}
$$

Related multiplication rule for two complex numbers by De Moivre is $z_{1} z_{2}=r_{1} r_{2}\left(\cos \left(\theta_{1}+\theta_{2}\right)+i \sin \left(\theta_{1}+\theta_{2}\right)\right)$ ([1], p. 14 \& [2], p. 13).

\section{Example}

Square of a number $1 \cos \theta_{1}+2 \sin \theta_{1}$ where $\theta_{1}=\tan ^{-1}(1 / 2)$ here angle $\theta_{1}=63.43495$ and $R_{1}=2.236068$ for first number and since it is a product of same number (i.e. square of a number) angle and $R$ are same, so product is equal to

$$
\begin{aligned}
& {[2.236068 \times 2.236068 \times \cos (126.8699)] \cos \theta_{3}+[2.236068 \times 2.236068 \times \sin (126.8699)] \sin \theta_{3}} \\
& {[2.236068 \times 2.236068 \times(-0.6)] \cos \theta_{3}+[2.236068 \times 2.236068 \times 0.8] \sin \theta_{3}}
\end{aligned}
$$


This is equal to

$$
-3 \cos \theta_{3}+4 \sin \theta_{3} \text { where } \theta_{3}=\tan ^{-1}(-3 / 4)
$$

This is identical to square of complex number $(1+2 i)$.

\section{Division of CNWOI}

There are two CNWOI

$$
a \cos \theta_{1}+b \sin \theta_{1} \text { where } \theta_{1}=\tan ^{-1}(a / b) \text { and } c \cos \theta_{2}+d \sin \theta_{2} \text { where } \theta_{2}=\tan ^{-1}(c / d)
$$

Then for division of $a \cos \theta_{1}+b \sin \theta_{1} / c \cos \theta_{2}+d \sin \theta_{2}$.

First find $R$ and angle $\theta$ for both numbers as did in multiplication calculation, then the division is equal to

$$
\begin{aligned}
& {\left[\left(R_{1} / R_{2}\right) \cos \left(\theta_{1}-\theta_{2}\right)\right] \cos \theta_{3}+\left[\left(R_{1} / R_{2}\right) \sin \left(\theta_{1}-\theta_{2}\right)\right] \sin \theta_{3}} \\
& \text { where } \theta_{3}=\tan ^{-1}\left(\left(R_{1} / R_{2}\right) \cos \left(\theta_{1}-\theta_{2}\right) /\left(R_{1} / R_{2}\right) \sin \left(\theta_{1}-\theta_{2}\right)\right)
\end{aligned}
$$

Related division rule for two complex number by De Moivre is $z_{1} / z_{2}=r_{1} / r_{2}\left(\cos \left(\theta_{1}-\theta_{2}\right)+i \sin \left(\theta_{1}-\theta_{2}\right)\right)$. ([1], $14 \&$ [2], p. 14).

\section{Example}

Division of two CNWOI

$$
1 \cos \theta_{1}+2 \sin \theta_{1} \text { where } \theta_{1}=\tan ^{-1}(1 / 2)
$$

So angle $\theta_{1}=63.43495$ and $R_{1}=2.236068$ for first number

$$
2 \cos \theta_{2}+3 \sin \theta_{2} \text { where } \theta_{2}=\tan ^{-1}(2 / 3)
$$

So angle $\theta_{2}=56.30993$ and $R_{2}=3.60551$ for second number.

Now $1 \cos \theta_{2}+2 \sin \theta_{2}$ divided by $2 \cos \theta_{2}+3 \sin \theta_{2}$ is equal to

$\left(R_{1} / R_{2}\right) \cos \left(\theta_{1}-\theta_{2}\right) \cos \theta_{3}+\left(R_{1} / R_{2}\right) \sin \left(\theta_{1}-\theta_{2}\right) \sin \theta_{3}$

$[2.236068 / 3.60551 \times \cos (63.43495-56.30993)] \cos \theta_{3}+[2.236068 / 3.60551 \times \sin (63.43495-56.30993)] \sin \theta_{3}$

$[0.620174 \times \cos (7.125016)] \cos \theta_{3}+[0.620174 \times \sin (7.125016)] \sin \theta_{3}$

$[0.620174 \times 0.992278] \cos \theta_{3}+[0.620174 \times 0.124035] \sin \theta_{3}$

$0.615385 \cos \theta_{3}+0.076923 \sin \theta_{3}$

This is equal to

$$
0.615385 \cos \theta_{3}+0.076923 \sin \theta_{3} \text { where } \theta_{3}=\tan ^{-1}(0.615385 / 0.076923)
$$

This is identical to the division of above complex numbers using imaginary number i, i.e. equal to $8 / 13+1 / 13 i$.

\section{Proof of Eluer Formula $\mathrm{e}^{\mathrm{i} \theta}=\cos (\theta)+i \sin (\theta)$ [1]-[4]}

Let $a+b i$ is any complex number

Its equivalent complex number without imaginary number $i$ (CNWOI) is

$$
a \cos (\theta)+b \sin (\theta) \text { where } \theta=\tan ^{-1}(a / b)
$$

Now $[\mathrm{e}]^{a \cos (\theta)+b \sin (\theta)}=[\mathrm{e}]^{a \cos \left(\theta_{1}\right)}[\mathrm{e}]^{b \sin \left(\theta_{2}\right)}$ since we have parted it into two complex numbers, there are two cases each for these two parted CNWOI

For first CNWOI $\theta_{1}$ may be 0 deg or 180 deg depend on the +ve or -ve value of " $a$ " and its $R$ is equal to ab- 
solute value of " $a$ ". Similarly for second CNWOI $\theta_{2}$ may be 90 deg or 270 deg depend on the +ve or -ve value of " $b$ " and its $R$ is equal to absolute value of " $b$ ", solving these cases one by one we get

For first CNWOI if $\theta_{1}$ is equal to 0 deg i.e. " $a$ " is any positive number on $\mathrm{X}$ axis, then

$$
[\mathrm{e}]^{a \cos (0)}=\left\{1+a \cos (0) / 1 !+(a \cos (0))^{2} / 2 !+(a \cos (0))^{3} / 3 !+(a \cos (0))^{4} / 4 !+\cdots\right\}
$$

Using product rule of CNWOI for terms with power greater than one and taking $R$ is equal to absolute value of " $a$ " we get

$$
\begin{aligned}
{[\mathrm{e}]^{a \cos (0)}=} & \left\{1+a \cos (0) / 1 !+\left\{\left[a^{2} \cos (2 \times 0)\right] \cos \left(\theta_{1}\right)+\left[a^{2} \sin (2 \times 0)\right] \sin \left(\theta_{1}\right)\right\} / 2 !\right. \\
& +\left\{\left[a^{3} \cos (3 \times 0)\right] \cos \left(\theta_{2}\right)+\left[a^{3} \sin (3 \times 0)\right] \sin \left(\theta_{2}\right)\right\} / 3 ! \\
& \left.+\left\{\left[a^{4} \cos (4 \times 0)\right] \cos \left(\theta_{3}\right)+\left[a^{4} \sin (4 \times 0)\right] \sin \left(\theta_{3}\right)\right\} / 4 !+\cdots\right\} \\
= & \left\{1+a \cos (0) / 1 !+a^{2} \cos \left(\theta_{1}\right) / 2 !+a^{3} \cos \left(\theta_{2}\right) / 3 !+a^{4} \cos \left(\theta_{3}\right) / 4 !+\cdots\right\}
\end{aligned}
$$

And using addition rule of CNWOI

$$
\left[1+a / 1 !+a^{2} / 2 !+a^{3} / 3 !+a^{4} / 4 !+\cdots\right] \cos (\alpha)=\mathrm{e}^{a} \cos (\alpha)
$$

So if " $a$ " is any positive number on $\mathrm{X}$ axis

$$
[\mathrm{e}]^{a \cos \left(\theta_{1}\right)}=\mathrm{e}^{a} \cos (\alpha)
$$

It is same as complex number with imaginary number $i$.

Now if $\theta_{1}$ is equal to 180 deg i.e. " $a$ " is any negative number on $\mathrm{X}$ axis then

$$
[\mathrm{e}]^{-a \cos (180)}=\left[1-a \cos (180) / 1 !+(-a \cos (180))^{2} / 2 !+(-a \cos (180))^{3} / 3 !+(-a \cos (180))^{4} / 4 !+\cdots\right]
$$

Using product rule of CNWOI for terms with power greater than one and talking $R$ is equal to absolute value of " $a$ " we get

$$
\begin{aligned}
{[\mathrm{e}]^{-a \cos (180)}=} & \left\{1-a \cos (180) / 1 !+\left\{\left[a^{2} \cos (2 \times 180)\right] \cos \left(\theta_{1}\right)+\left[a^{2} \sin (2 \times 180)\right] \sin \left(\theta_{1}\right)\right\} / 2 !\right. \\
& +\left\{\left[a^{3} \cos (3 \times 180)\right] \cos \left(\theta_{2}\right)+\left[a^{3} \sin (3 \times 180)\right] \sin \left(\theta_{2}\right)\right\} / 3 ! \\
& \left.+\left\{\left[a^{4} \cos (4 \times 180)\right] \cos \left(\theta_{3}\right)+\left[a^{4} \sin (4 \times 180)\right] \sin \left(\theta_{3}\right)\right\} / 4 !+\cdots\right\} \\
= & \left\{1-a \cos (180) / 1 !+a^{2} \cos \left(\theta_{1}\right) / 2 !-a^{3} \cos \left(\theta_{2}\right) / 3 !+a^{4} \cos \left(\theta_{3}\right) / 4 !+\cdots\right\}
\end{aligned}
$$

Using addition rule of CNWOI

$$
\left[1-a / 1 !+a^{2} / 2 !-a^{3} / 3 !+a^{4} / 4 !+\cdots\right] \cos (\alpha)=\mathrm{e}^{-a} \cos (\alpha)
$$

So if " $a$ " is any negative number on $\mathrm{X}$ axis

$$
[\mathrm{e}]^{-a \cos \left(\theta_{1}\right)}=\mathrm{e}^{-a} \cos (\alpha)
$$

It is same as complex number with imaginary number $i$.

Now for second CNWOI if $\theta_{2}$ is equal to 90 deg i.e. " $b$ " is any positive number on $\mathrm{Y}$ axis then

$$
[\mathrm{e}]^{b \sin (90)}=\left[1+b \sin (90) / 1 !+(b \sin (90))^{2} / 2 !+(b \sin (90))^{3} / 3 !+(b \sin (90))^{4} / 4 !+\cdots\right]
$$

Using product rule of CNWOI for terms with power greater than one and talking $R$ is equal to absolute value of " $b$ " we get 


$$
\begin{aligned}
{[\mathrm{e}]^{b \sin (90)}=} & \left\{1+b \sin (90) / 1 !+\left\{\left[b^{2} \cos (2 \times 90)\right] \cos \left(\theta_{1}\right)+\left[b^{2} \sin (2 \times 90)\right] \sin \left(\theta_{1}\right)\right\} / 2 !\right. \\
& +\left\{\left[b^{3} \cos (3 \times 90)\right] \cos \left(\theta_{2}\right)+\left[b^{3} \sin (3 \times 90)\right] \sin \left(\theta_{2}\right)\right\} / 3 ! \\
& \left.+\left\{\left[b^{4} \cos (4 \times 90)\right] \cos \left(\theta_{3}\right)+\left[b^{4} \sin (4 \times 90)\right] \sin \left(\theta_{3}\right)\right\} / 4 !+\cdots\right\} \\
{[\mathrm{e}]^{b \sin (90)}=} & \left\{1+b \sin (90) / 1 !+\left\{\left[b^{2} \cos (180)\right] \cos \left(\theta_{1}\right)+\left[b^{2} \sin (180)\right] \sin \left(\theta_{1}\right)\right\} / 2 !\right. \\
& +\left\{\left[b^{3} \cos (270)\right] \cos \left(\theta_{2}\right)+\left[b^{3} \sin (270)\right] \sin \left(\theta_{2}\right)\right\} / 3 ! \\
& \left.+\left\{\left[b^{4} \cos (360)\right] \cos \left(\theta_{3}\right)+\left[b^{4} \sin (360)\right] \sin \left(\theta_{3}\right)\right\} / 4 !+\cdots\right\} \\
= & {\left[1+b \sin (90) / 1 !-b^{2} \cos \left(\theta_{1}\right) / 2 !-b^{3} \sin \left(\theta_{2}\right) / 3 !+b^{4} \cos \left(\theta_{3}\right) / 4 !+\cdots\right] }
\end{aligned}
$$

Using addition rule of CNWOI

$$
\begin{aligned}
& =\left[1-b^{2} / 2 !+b^{4} / 4 !+\cdots\right] \cos (\alpha)+\left[b / 1 !-b^{3} / 3 !+\cdots\right] \sin (\alpha) \\
& =[\cos (b)] \cos (\alpha)+[\sin (b)] \sin (\alpha)
\end{aligned}
$$

So if " $b$ " is any positive number on $\mathrm{Y}$ axis

$$
[\mathrm{e}]^{b \sin \left(\theta_{2}\right)}=[\cos (b)] \cos (\alpha)+[\sin (b)] \sin (\alpha)
$$

It is same as complex number with imaginary number, that is $[\mathrm{e}]^{i b}=\cos (b)+i \sin (b)$.

Remember that a complex number with $i$ is equal to $a+i b$ and its equivalent CNWOI is $a \cos (\alpha)+b \sin (\alpha)$. Now if $\theta_{2}$ is equal to 270 deg i.e. " $b$ " is any negative number on $y$ axis then

$$
[\mathrm{e}]^{-b \sin (270)}=\left[1-b \sin (270) / 1 !+(-b \sin (270))^{2} / 2 !+(-b \sin (270))^{3} / 3 !+(-b \sin (270))^{4} / 4 !+\cdots\right]
$$

Using product rule of CNWOI for terms with power greater than one and talking $R$ is equal to absolute value of " $b$ " we get

$$
\begin{aligned}
{[\mathrm{e}]^{-b \sin (270)}=} & \left\{1-b \sin (270) / 1 !+\left\{\left[b^{2} \cos (2 \times 270)\right] \cos \left(\theta_{1}\right)+\left[b^{2} \sin (2 \times 270)\right] \sin \left(\theta_{1}\right)\right\} / 2 !\right. \\
& +\left\{\left[b^{3} \cos (3 \times 270)\right] \cos \left(\theta_{2}\right)+\left[b^{3} \sin (3 \times 270)\right] \sin \left(\theta_{2}\right)\right\} / 3 ! \\
& \left.+\left\{\left[b^{4} \cos (4 \times 270)\right] \cos \left(\theta_{3}\right)+\left[b^{4} \sin (4 \times 270)\right] \sin \left(\theta_{3}\right)\right\} / 4 !+\cdots\right\} \\
{[\mathrm{e}]^{-b \sin (270)}=} & \left\{1-b \sin (270) / 1 !+\left\{\left[b^{2} \cos (540)\right] \cos \left(\theta_{1}\right)+\left[b^{2} \sin (540)\right] \sin \left(\theta_{1}\right)\right\} / 2 !\right. \\
& +\left\{\left[b^{3} \cos (810)\right] \cos \left(\theta_{2}\right)+\left[b^{3} \sin (810)\right] \sin \left(\theta_{2}\right)\right\} / 3 ! \\
& \left.+\left\{\left[b^{4} \cos (1080)\right] \cos \left(\theta_{3}\right)+\left[b^{4} \sin (1080)\right] \sin \left(\theta_{3}\right)\right\} / 4 !+\cdots\right\} \\
{[\mathrm{e}]^{-b \sin (270)=}=} & \left\{1-b \sin (270) / 1 !+\left\{\left[b^{2} \cos (180)\right] \cos \left(\theta_{1}\right)+\left[b^{2} \sin (180)\right] \sin \left(\theta_{1}\right)\right\} / 2 !\right. \\
& +\left\{\left[b^{3} \cos (90)\right] \cos \left(\theta_{2}\right)+\left[b^{3} \sin (90)\right] \sin \left(\theta_{2}\right)\right\} / 3 ! \\
& \left.+\left\{\left[b^{4} \cos (360)\right] \cos \left(\theta_{3}\right)+\left[b^{4} \sin (360)\right] \sin \left(\theta_{3}\right)\right\} / 4 !+\cdots\right\} \\
= & {\left[1-b \sin (270) / 1 !-b^{2} \cos \left(\theta_{1}\right) / 2 !+b^{3} \sin \left(\theta_{2}\right) / 3 !+b^{4} \cos \left(\theta_{3}\right) / 4 !+\cdots\right] }
\end{aligned}
$$

Using addition rule of CNWOI

$$
\begin{aligned}
= & {\left[1-b^{2} / 2 !+b^{4} / 4 !+\cdots\right] \cos (\alpha)+\left[-b / 1 !+b^{3} / 3 !+\cdots\right] \sin (\alpha) } \\
& {\left[1-b^{2} / 2 !+b^{4} / 4 !+\cdots\right] \cos (\alpha)-\left[b / 1 !-b^{3} / 3 !+\cdots\right] \sin (\alpha) } \\
= & {[\cos (b)] \cos (\alpha)-[\sin (b)] \sin (\alpha) }
\end{aligned}
$$


So if " $b$ " is any negative number on $\mathrm{Y}$ axis

$$
[\mathrm{e}]^{-b \sin \left(\theta_{2}\right)}=[\cos (b)] \cos (\alpha)-[\sin (b)] \sin (\alpha)
$$

It is same as complex number with imaginary number $i$, that is $[\mathrm{e}]^{-i b}=\cos (b)-i \sin (b)$.

\section{De Moivre Theorem (for Finding Power of Complex Number)}

In complex number if $n$ is a positive integer, then

$$
[\cos (\theta)+i \sin (\theta)]^{n}=\cos (n \theta)+i \sin (n \theta) \quad \text { ([2], p. 15] }
$$

Proof of this theorem in complex number theory without " $i$ " (CNWOI) is as follows

Let $a+i b$ is any complex number

Its equivalent CNWOI is $[a \cos (\theta)+b \sin (\theta)]$ now we have to prove that

$$
[a \cos (\theta)+b \sin (\theta)]^{n}=c \cos (n \theta)+d \sin (n \theta)
$$

where $c=R^{n} \cos (n \theta) \& d=R^{n} \sin (n \theta)$ are the numbers came out after multiplying same CNWOI " $n$ " times and $\theta=\tan ^{-1}(c / d)$.

Formula for multiplication of two CNWOI is

$$
\left[R_{1} R_{2} \cos \left(\theta_{1}+\theta_{2}\right)\right] \cos \alpha+\left[R_{1} R_{2} \sin \left(\theta_{1}+\theta_{2}\right)\right] \sin \alpha
$$

where

$$
\alpha=\tan ^{-1}\left(\left[R_{1} R_{2} \cos \left(\theta_{1}+\theta_{2}\right)\right] /\left[R_{1} R_{2} \sin \left(\theta_{1}+\theta_{2}\right)\right]\right)
$$

Since we were multiplying same CNWOI " $n$ " times

$$
\begin{gathered}
R_{1}=R_{2}=R_{3}=\cdots=R_{n}=R \quad \text { (some modulus/radius associated with given CNWOI) } \\
\theta_{1}=\theta_{2}=\theta_{3}=\cdots=\theta_{n}=\theta \quad \text { (some angle associated with given CNWOI) }
\end{gathered}
$$

So product is

$$
\begin{aligned}
& {[a \cos (\theta)+b \sin (\theta)]^{n}=[R \times R \times \cdots \times R \cos (\theta+\theta+\cdots+\theta)] \cos \alpha+[R \times R \times \cdots \times R \sin (\theta+\theta+\cdots+\theta)] \sin \alpha} \\
& {[a \cos (\theta)+b \sin (\theta)]^{n}=\left[R^{n} \cos (n \theta)\right] \cos \alpha+\left[R^{n} \sin (n \theta)\right] \sin \alpha}
\end{aligned}
$$

where

$$
\alpha=\tan ^{-1}\left(R^{n} \cos (n \theta) / R^{n} \sin (n \theta)\right)
$$

$\alpha$ will be equal to $n \theta$.

Putting this in Equation (1) we get

$$
[a \cos (\theta)+b \sin (\theta)]^{n}=c \cos (n \theta)+d \sin (n \theta)
$$

where

$$
c=[R]^{n} \cos (n \theta) \text { and } d=[R]^{n} \sin (n \theta)
$$

\section{Example}

Let $6+8 i$ is any complex number. Now we find out its cube using De Moivre theorem of CNWOI i.e.

$(6+8 i)^{3}$ is equal to $[6 \cos (\theta)+8 \sin (\theta)]^{3}$ in CNWOI

$$
[a \cos (\theta)+b \sin (\theta)]^{n}=c \cos (n \theta)+d \sin (n \theta)
$$

$$
\theta=\tan ^{-1}(6 / 8)=53.1301
$$




$$
\begin{aligned}
& R=6 \cos (53.1301)+8 \sin (53.1301) \\
& R=6 \times 0.6+8 \times 0.8=3.6+6.4=10 \\
& R=10
\end{aligned}
$$

Now using De Moivre theorem

$$
\begin{aligned}
& {[6 \cos (\theta)+8 \sin (\theta)]^{3}=\left[(10)^{3} \cos (3 \times 53.1301)\right] \cos (\alpha)+\left[(10)^{3} \sin (3 \times 53.1301)\right] \sin (\alpha)} \\
& =[(1000) \cos (159.3903)] \cos (\alpha)+[(1000) \sin (159.3903)] \sin (\alpha) \\
& =[(1000)(-0.936)] \cos (\alpha)+[(1000)(0.352)] \sin (\alpha) \\
& =-936 \cos (\alpha)+352 \sin (\alpha) \\
& \alpha=\tan ^{-1}(-936 / 352)=159.3903=3 \theta \\
& {[6 \cos (\theta)+8 \sin (\theta)]^{3}=-936 \cos (3 \theta)+352 \sin (3 \theta)}
\end{aligned}
$$

This is equal to cube of $6+8 i$.

\section{De Moivre Theorem (for Finding Root of Complex Number)}

For finding root of complex number take compliment of De Moivre theorem (for finding power of CNWOI).

If $n$ is a positive integer, then $n^{\text {th }}$ root for any CNWOI $[a \cos (\theta)+b \sin (\theta)]$ is equal to

$$
[a \cos (\theta)+b \sin (\theta)]^{1 / n}=c \cos (\theta / n)+d \sin (\theta / n)
$$

where $c=R^{1 / n} \cos (\theta / n) \& d=R^{1 / n} \sin (\theta / n)$ and $\theta=\tan ^{-1}(c / d)=\theta / n$.

\section{Example}

Let $-936+i 352$ is any complex number. Now find out its cubic root using De Moivre theorem of CNWOI

$$
[a \cos (\theta)+b \sin (\theta)]^{1 / n}=c \cos (\theta / n)+d \sin (\theta / n)
$$

where

$$
\begin{gathered}
c=R^{1 / n} \cos (\theta / n) \& d=R^{1 / n} \sin (\theta / n) \text { and } \theta=\tan ^{-1}(c / d)=\theta / n \\
{[-936 \cos (\theta)+352 \sin (\theta)]^{1 / 3}}
\end{gathered}
$$

Here $\theta=\tan ^{-1}(-936 / 352)=159.3903$.

Putting value $\theta$ in CNWOI $-936 \cos (\theta)+352 \sin (\theta)$ we get $R$

$-936 \cos (159.3903)+352 \sin (159.3903)=-936 \times(-0.936)+352 \times(0.352)=876.096+123.904=1000$

$$
R=1000
$$

Putting this $R$ and $\theta$ in Equation (2) we get $c \& d$

$$
c=(1000)^{1 / 3} \cos (159.3903 / 3)=10 \times \cos (53.1301)=10 \times 0.6=6
$$

and

$$
d=(1000)^{1 / 3} \sin (159.3903 / 3)=10 \times \sin (53.1301)=10 \times 0.8=8
$$

Putting these values of $c \& d$ in Equation (1) we get

$$
[-936 \cos (159.3903)+352 \sin (159.3903)]^{1 / 3}=6 \cos (53.1301)+8 \sin (53.1301)
$$

This is a cubic root of complex number $-936+i 352$. 


\section{Solution of Quadratic Equation Using CNWOI}

Quadratic equations $\left(a x^{2}+b x+c=0\right)$ for which $b^{2}-4 a c<0$.

First find out $\theta$ (i.e. angle/argument) and $R$ (i.e. modulus/radius), which are equal to

$$
\begin{gathered}
\theta=\tan ^{-1}[-b / 2 a] /\left[\left(a b s\left(b^{2}-4 a c\right)\right) / 2 a\right] \\
R=(-b / 2 a) \cos (\theta)+\left(\sqrt{a b s\left(b^{2}-4 a c\right)}\right) / 2 a \sin (\theta)
\end{gathered}
$$

Hence roots for quadratic equation are

$$
R(\cos (\theta)+\sin (\theta)) \text { and } R(\cos (\theta)-\sin (\theta))
$$

\subsection{Example 1}

Let $x^{2}+x+1=0$ for which $b^{2}-4 a c<0$, then $(-b / 2 a)=-1 / 2=-0.5$ and $\left(\sqrt{a b s\left(b^{2}-4 a c\right)}\right) / 2 a=0.8660254$.

$\theta$ i.e. angle/argument and $R$ i.e. modulus/radius are equal to

$$
\begin{gathered}
\theta=\tan ^{-1}\left((-b / 2 a) /\left(\sqrt{a b s\left(b^{2}-4 a c\right)}\right) / 2 a\right)=\tan ^{-1}(-0.5 / 0.8660254)=120 \\
R=(-b / 2 a) \cos (\theta)+\left(\sqrt{a b s\left(b^{2}-4 a c\right)}\right) / 2 a \sin (\theta)=-0.5 \cos (120)+0.8660254 \sin (120) \\
=(-0.5) \times(-0.5)+0.8660254 \times 0.8660254=0.25+0.75=1
\end{gathered}
$$

Hence roots for quadratic equation are

$$
(\cos (120)+\sin (120)) \&(\cos (120)-\sin (120))
$$

Putting first root in quadratic equation we get

$$
\begin{aligned}
& {[\cos (120)+\sin (120)]^{2}+\cos (120)+\sin (120)+1=[\cos (240)+\sin (240)]+\cos (120)+\sin (120)+1} \\
& =-0.5+(-0.8660254)+(-0.5)+0.8660254+1=-1+0+1=0
\end{aligned}
$$

Putting second root in quadratic equation we get

$$
\begin{aligned}
& {[\cos (120)-\sin (120)]^{2}+\cos (120)-\sin (120)+1=[\cos (240)-\sin (240)]+\cos (120)-\sin (120)+1} \\
& =-0.5+0.8660254+(-0.5)-0.8660254+1=-1+0+1=0
\end{aligned}
$$

\subsection{Example 2}

Let $x^{2}-10 x+40=0$ for which $b^{2}-4 a c<0$, then $(-b / 2 a)=5$ and $\left(\sqrt{a b s\left(b^{2}-4 a c\right)}\right) / 2 a=3.8729833$.
$\theta$ i.e. angle/argument and $R$ i.e. modulus/radius are equal to

$$
\begin{gathered}
\theta=\tan ^{-1}\left((-b / 2 a) /\left(\sqrt{a b s\left(b^{2}-4 a c\right)}\right) / 2 a\right)=\tan ^{-1}(5 / 3.8729833)=37.761244 \\
R=(-b / 2 a) \cos (\theta)+\left(\sqrt{a b s\left(b^{2}-4 a c\right)}\right) / 2 a \sin (\theta)=5 \cos (37.761244)+3.8729833 \sin (37.761244) \\
=5 \times 0.7905694+3.8729833 \times 0.6123724=3.9528471+2.3717082=6.3245553
\end{gathered}
$$

Hence roots for quadratic equation are

$$
6.3245553 \times(\cos (37.761244)+\sin (37.761244)) \& 6.3245553 \times(\cos (37.761244)-\sin (37.761244))
$$

Putting first root in quadratic equation we get 


$$
\begin{aligned}
& {[6.3245553(\cos (37.761244)+\sin (37.761244))]^{2}+(-10) \times 6.3245553} \\
& \times(\cos (37.761244)+\sin (37.761244))+40 \\
& =40 \times[\cos (75.522488)+\sin (75.522488)]+(-63.245553) \times(\cos (37.761244)+\sin (37.761244))+40 \\
& =10+38.729833+(-50)+(-38.729833)+40=-40+40=0
\end{aligned}
$$

Putting second root in quadratic equation we get

$$
\begin{aligned}
& {[6.3245553(\cos (37.761244)-\sin (37.761244))]^{2}+(-10) \times 6.3245553} \\
& \times(\cos (37.761244)-\sin (37.761244))+40 \\
& =40 \times[\cos (75.522488)-\sin (75.522488)]+(-63.245553) \times(\cos (37.761244)-\sin (37.761244))+40 \\
& =10-38.729833+(-50)-(-38.729833)+40=-40+40=0
\end{aligned}
$$

\subsection{Example 3}

Let $3 x^{2}-4 x+2=0$ for which $b^{2}-4 a c<0$, then $(-b / 2 a)=0.6666667$ and $\left(\sqrt{a b s\left(b^{2}-4 a c\right)}\right) / 2 a=0.4714045$.

$\theta$ i.e. angle/argument and $R$ i.e. modulus/radius are equal to

$$
\begin{gathered}
\theta=\tan ^{-1}\left((-b / 2 a) /\left(\sqrt{a b s\left(b^{2}-4 a c\right)}\right) / 2 a\right)=\tan ^{-1}(0.6666667 / 0.4714045)=35.26439 \\
R=(-b / 2 a) \cos (\theta)+\left(\sqrt{a b s\left(b^{2}-4 a c\right)}\right) / 2 a \sin (\theta)=0.6666666 \cos (35.26439)+0.4714045 \sin (35.26439) \\
=0.6666667 \times 0.8164966+0.4714045 \times 0.5773503=0.5443311+0.2721655=0.8164966
\end{gathered}
$$

Hence roots for quadratic equation are

$$
0.8164966 \times(\cos (35.26439)+\sin (35.26439)) \& 0.8164966 \times(\cos (35.26439)-\sin (35.26439))
$$

Putting first root in quadratic equation we get

$$
\begin{aligned}
& 3 \times[0.8164966 \times(\cos (35.26439)+\sin (35.26439))]^{2}+(-4) \times 0.8164966 \\
& \times(\cos (35.26439)+\sin (35.26439))+2 \\
& =0.6666667+1.8856181+(-2.6666667)-1.8856181+2=-2+2=0
\end{aligned}
$$

Putting second root in quadratic equation we get

$$
\begin{aligned}
& 3 \times[0.8164966 \times(\cos (35.26439)-\sin (35.26439))]^{2}+(-4) \times 0.8164966 \\
& \times(\cos (35.26439)-\sin (35.26439))+2 \\
& =0.6666667-1.8856181+(-2.6666667)-(-1.8856181)+2=-2+2=0
\end{aligned}
$$

\subsection{Example 4}

Let $-4 x^{2}+2 x-7=0$ for which $b^{2}-4 a c<0$, then $(-b / 2 a)=0.25$ and $\left(\sqrt{a b s\left(b^{2}-4 a c\right)}\right) / 2 a=-1.2990381$.

$\theta$ i.e. angle/argument and $R$ i.e. modulus/radius are equal to

$$
\theta=\tan ^{-1}\left((-b / 2 a) /\left(\sqrt{a b s\left(b^{2}-4 a c\right)}\right) / 2 a\right)=\tan ^{-1}(0.25 /-1.2990381)=-79.106605=280.89339
$$




$$
\begin{aligned}
R & =(-b / 2 a) \cos (\theta)+\left(\sqrt{a b s\left(b^{2}-4 a c\right)}\right) / 2 a \sin (\theta)=0.25 \cos (280.89339)+(-1.2990381) \sin (280.89339) \\
& =0.25 \times 0.1889822+(-1.2990381) \times(-0.9819805)=0.0472456+1.2756301=1.3228757
\end{aligned}
$$

Hence roots for quadratic equation are

$$
1.3228757[\cos (280.89339)+\sin (280.89339)] \& 1.3228757[\cos (280.89339)-\sin (280.89339)]
$$

Putting first root in quadratic equation we get

$$
\begin{aligned}
& -4 \times[1.3228757 \times(\cos (280.89339)+\sin (280.89339))]^{2}+2 \times 1.3228757 \\
& \times[\cos (280.89339)+\sin (280.89339)]-7 \\
& =6.5+2.5980762+0.5-2.5980762-7=7-7=0
\end{aligned}
$$

Putting second root in quadratic equation we get

$$
\begin{aligned}
& -4 \times[1.3228757 \times(\cos (280.89339)-\sin (280.89339))]^{2}+2 \times 1.3228757 \\
& \times[\cos (280.89339)-\sin (280.89339)]-7 \\
& =6.5-2.5980762+0.5+2.5980762-7=7-7=0
\end{aligned}
$$

\subsection{Example 5}

Let $x^{2}+1=0$ for which $b^{2}-4 a c<0$, then $(-b / 2 a)=0$ and $\left(\sqrt{a b s\left(b^{2}-4 a c\right)}\right) / 2 a=1$.
First find out $\theta$ i.e. angle/argument and $R$ i.e. modulus/radius which are equal to

$$
\begin{gathered}
\theta=\tan ^{-1}\left((-b / 2 a) /\left(\sqrt{a b s\left(b^{2}-4 a c\right)}\right) / 2 a\right)=\tan ^{-1}(0 / 1)=90 \\
R=(-b / 2 a) \cos (\theta)+\left(\sqrt{a b s\left(b^{2}-4 a c\right)}\right) / 2 a \sin (\theta)=0 \cos (90)+1 \sin (90)=1
\end{gathered}
$$

Hence roots for quadratic equation are

$$
1 \times[\cos (90)+\sin (90)] \& 1 \times[\cos (90)-\sin (90)]
$$

Putting first root in quadratic equation we get

$$
\begin{aligned}
& {[1 \times(\cos (90)+\sin (90))]^{2}+1} \\
& {[1 \times(\cos (180)+\sin (180))]+1=-1+0+1=0}
\end{aligned}
$$

Putting second root in quadratic equation we get

$$
\begin{aligned}
& {[1 \times(\cos (90)-\sin (90))]^{2}+1} \\
& {[1 \times(\cos (180)-\sin (180))]+1=-1-0+1=0}
\end{aligned}
$$

\section{About Square Root of Negative Real Number}

Imaginary number $i=\sqrt{-1}=(-1)^{1 / 2}$ where -1 is a negative real number. Its modulus or radius is equal to 1 and angle is equal to 180 degrees, so using De Moivre theorem of CNWOI

$$
[a \cos (\theta)+b \sin (\theta)]^{1 / n}=c \cos (\theta / n)+d \sin (\theta / n)
$$

where $c=R^{1 / n} \cos (\theta / n)$ and $d=R^{1 / n} \sin (\theta / n)$ and $\theta=\tan ^{-1}(c / d)=\theta / n$ since $(-1)^{1 / 2}$ has $R=1$ and $\theta=$ 180 deg calculating $c \& d$ using above formula 


$$
\begin{aligned}
& c=(1)^{1 / 2} \cos (180 / 2) \\
& c=\cos (90)=0
\end{aligned}
$$

and

$$
\begin{aligned}
& d=(1)^{1 / 2} \sin (180 / 2) \\
& d=\sin (90)=1
\end{aligned}
$$

So putting values of $c \& d$ in above formula we get

$$
\begin{aligned}
& (-1)^{1 / 2}=0 \times \cos (90)+1 \times \sin (90) \\
& (-1)^{1 / 2}=0+\sin (90) \\
& (-1)^{1 / 2}=\sin (90)
\end{aligned}
$$

It is equivalent to the graphical position and value of imaginary number " $i$ ".

So the square root of negative number is equal to the square root of same positive real number but with an angle of a 90 deg to real line.

Why the square root of negative number is at 90 deg to real line? It is in the roots of rules of multiplication where

$$
\begin{gathered}
\text { Positive } \times \text { Positive }=\text { Positive } \\
\text { Positive } \times \text { Negative }=\text { Negative } \\
\text { Negative } \times \text { Negative }=\text { Positive }
\end{gathered}
$$

These rules build a limitation when we come across a situation where we need square root of negative number. There is no negative number multiplied by itself will give a negative number.

Complex number is an answer to this limitation. Complex number is nothing but an interaction of two mutually opposite number systems, particularly in multiplication rules. Their individual and combine behavior under multiplication operation are given below.

\subsection{When Only X Axis Is Considered It Gives}

$$
\text { Positive } \times \text { Negative }=\text { Negative }
$$

i.e. $a \cos (0) \times-b \cos (180)$ using multiplication rule of CNWOI is equal to [-abcos(180)]

and

$$
\text { Negative } \times \text { Negative }=\text { Positive }
$$

i.e. $-a \cos (180) \times-b \cos (180)$ using multiplication rule of CNWOI is equal to $[a b \cos (0)]$.

\subsection{When Only Y Axis Is Considered It Gives}

$$
\text { Positive } \times \text { Negative }=\text { Positive }
$$

i.e. $a \sin (90) \times-b \sin (270)$ using multiplication rule of CNWOI is equal to $[a b \cos (0)]$

and

$$
\text { Negative } \times \text { Negative }=\text { Negative }
$$

i.e. $-a \sin (270) \times-b \sin (270)$ using multiplication rule of CNWOI is equal to [ $-a b \cos (180)]$.

\subsection{But Jointly They Give Back Traditional Results Such As}

$$
\text { Positive } \times \text { Negative }=\text { Negative }
$$

i.e. $a \cos (0) \times-b \sin (270)$ using product rule of CNWOI is equal to $-a b \sin (270)$ or 
$-a \cos (180) \times b \sin (90)$ using product rule of CNWOI is equal to $-a b \sin (270)$

and

$$
\text { Negative } \times \text { Negative }=\text { Positive }
$$

i.e. $-a \cos (180) \times-b \sin (270)$ using product rule of CNWOI is equal to $a b \sin (90)$.

\section{Final Reflection}

As said earlier in graphical representation of complex number, the length of $i$ shown on vertical axis is equal to 1 ; it is a contradiction since value of imaginary number $i$ is not known. Also division process is not smooth, for dividing one complex number with other needs to multiply it by conjugate of divisor. But in CNWOI theory it looks smooth and exactly opposite to the process of multiplication.

If addition, subtraction, multiplication, division, power and root can be found/calculated without imaginary number " $i$ ", presented in this paper, so there is nothing mystical or imaginary about imaginary number " $i$ ”. Complex numbers exist without imaginary number “ $i$ ”.

\section{References}

[1] Narayan, S. and Mittal, P.K. (2005) Theory of Functions of a Complex Variable. S. Chand \& Company Ltd., New Delhi.

[2] Schneider, C. (2011) De Moivre’s Theorem - A Literature and Curriculum Project on Roots, Powers, and Complex Numbers. Department of Mathematics and Statistics, Portland State University.

[3] Bogomolny, A. (1996-2014) Cut the Knot! An Interactive Column Using Java Applets. http://www.cut-the-knot.org/arithmetic/algebra/ComplexNumbers.shtml

[4] Cain, G. (1999-2014) Complex Analysis. http://people.math.gatech.edu/ cain/winter99/complex.html 